\title{
CONDICIONES DE IDENTIDAD PARA UNIVERSALES TRASCENDENTES ${ }^{1}$ \\ Identity Conditions for transcendent Universals
}

José Tomás Alvarado Marambio*

Resumen

En este trabajo se pretende argumentar que no es incoherente atribuir a los universales trascendentes condiciones de identidad fundadas en los poderes causales que tales universales confieren a los objetos que los instancian. Luego de explicar de manera sucinta algunas nociones cruciales de metafísica de propiedades, se indica cómo las condiciones de identidad para propiedades han tendido a diferenciarse entre las llamadas "propiedades abundantes" y las "propiedades escasas".

Palabras clave: Universales, universales inmanentes, universales trascendentes, condiciones de identidad, poderes causales.

Abstract

In this work it is argued that it is not incoherent to attribute conditions of identity founded on causal powers to transcendent universals. After explaining some crucial notions of metaphysics of properties, it is said how conditions of identity for properties have been differentiated between so called "abundant properties" and "sparse properties".

Key words: Universals, Inmanent Universals, Transcendent Universals, Conditions of Identity, Causal Powers.

Se ha distinguido entre teorías de propiedades "abundantes" y "escasas" (Lewis, 1983:10-19), entendiéndose por "propiedad" una determinación, característica o rasgo de algo, típicamente, de un objeto individual. Han existido muchas formas en que tales propiedades han sido concebidas. Las teorías de propiedades "abundantes" postulan una multitud enorme de entidades. Por ejemplo, propiedades como el correlato semántico de cada uno de los infinitos predicados de nuestros lenguajes naturales o, bien, propiedades asociadas con todos los conjuntos que puedan formarse tomando ciertos objetos como elementos. Las teorías de propiedades "escasas", en cambio, postulan propiedades como entidades que no están correlacionadas, al menos no inmediatamente, con los predicados de algún lenguaje y cuya existencia no depende - como en las teorías de propiedades "abundantes"- básicamente de reflexión a priori, sino de investigación empírica sobre los respectivos objetivos de semejanza o desemejanza entre objetos, que resultan relevantes para las conexiones causales en las que los objetos que

1 Este trabajo ha sido redactado en ejecución del proyecto de investigación Fondecyt $\mathrm{N}^{\circ} 1090002$ (Conicyt, Chile). 
instancian tales propiedades puedan entrar. Estas propiedades "escasas" serán las que entren en las leyes naturales, cuyo descubrimiento es, por supuesto, una cuestión $a$ posteriori. Ha sido frecuente asociar las teorías de propiedades "escasas" con teorías de universales inmanentes, esto es, universales que deben estar instanciados para existir, y las teorías de propiedades "abundantes" con teorías de universales trascendentes, esto es, universales que no requieren estar instanciados para existir. Estas dos grandes concepciones, también, tienen asociadas especificaciones diferentes de las condiciones de identidad para propiedades. Las teorías de propiedades "escasas" tienden a identificar las propiedades con roles causales, mientras que las teorías de propiedades "abundantes" tienden a identificar las propiedades con ítems proposicionales, capaces de especificar actitudes intencionales.

Hay aquí, por supuesto, muchos aspectos que requieren matización. Nuestro objetivo es mostrar cómo es que esta imagen general debe ser tomada con especial cautela. En particular, se pretende mostrar cómo es que perfectamente los universales trascendentes pueden tener condiciones de identidad dadas por la relevancia causal de tales propiedades, aun suponiendo que ellos no entran en relaciones causales (al menos, no directamente). Será necesario, antes de considerar esta cuestión derechamente, hacer algunas precisiones conceptuales previas.

\section{UNIVERSALES, TROPOS, CLASES, ESCASEZ, ABUNDANCIA}

La cuestión aquí es restringir la discusión al contraste entre universales inmanentes y trascendentes, pero hay una pluralidad de otras opciones en metafísica de propiedades, donde la distinción general entre concepciones de propiedades "escasas"/"abundantes" puede aplicarse. Estipulamos que una "propiedad" es una determinación, carácter o rasgo de un objeto que puede ser distinguida como una entidad diferente del objeto que la posee. ${ }^{2}$ La relación que se da entre un objeto y su propiedad es denominada "instanciación". Las teorías que niegan la existencia de propiedades como entidades diferentes de los objetos que las instancian son llamadas "nominalistas". Por ejemplo, en las teorías nominalistas más sofisticadas, las propiedades son eliminadas por clases de objetos posibles (esto es, objetos en diferentes mundos posibles) o por objetos que conforman clases seleccionadas por la semejanza perfecta entre sus elementos. En el primer caso, las clases de objetos posibles pueden cumplir las funciones teóricas requeridas por las propiedades "abundantes" y también por las propiedades "escasas". En

\footnotetext{
${ }^{2}$ Esta estipulación, entonces, deja fuera - como teorías que no admiten la existencia de propiedades - a las teorías que postulan que los rasgos o caracteres de las cosas son clases, sean o no sean de semejanza. Lewis, sin embargo, postula clases de objetos actuales y meramente posibles para cumplir las funciones exigidas de las propiedades y, de hecho, denomina como "propiedades" a tales clases. Cfr., Lewis (1983:10); Rodriguez-Pereyra (2002:14-21). Esta diferencia terminológica no será relevante para lo que sigue.
} 
efecto, entre las incontables clases de objetos posibles que pueden ser formadas de acuerdo con los axiomas estándar de la teoría de conjuntos, pueden seleccionarse algunas que conformen "clases naturales", en tanto sus elementos tengan, entre sí, una relación primitiva de semejanza relevante para la descripción de los respectos objetivos de la realidad, tal como estos respectos se recogen en nuestras mejores teorías científicas. ${ }^{3}$ En el segundo caso, las propiedades no son eliminadas por clases, entre las cuales pueden, luego, seleccionarse las que posean objetos con semejanza perfecta, sino directamente por tal relación de semejanza perfecta. Aquí, tal como puede apreciarse, el nominalista de semejanza pretende ofrecer entidades que cumplan las funciones de propiedades "escasas". 4

Una vez que se acepta la existencia de propiedades como entidades diferentes de los objetos que las instancian, existe la alternativa de concebir estas propiedades como entidades individuales y no de carácter universal, esto es, tropos. Usualmente, los defensores de tropos han preferido una concepción de propiedades "escasas" en donde los tropos son relata de conexiones causales y son las entidades descritas en las leyes naturales, ofreciendo respectos objetivos de semejanza y desemejanza (Cfr., Campbell, 1981:480-481). No es incoherente, sin embargo, una concepción de tropos "abundantes" donde cada predicado atribuido a un objeto individual designe un tropo diferente. La distinción entre teorías de propiedades "escasas" y "abundantes", sin embargo, se ha mostrado más marcadamente en relación con las teorías de universales. Un universal es una propiedad que, en virtud de su naturaleza, puede encontrarse instanciada en una pluralidad de casos. Tradicionalmente, se ha diferenciado entre universales inmanentes o aristotélicos que solamente existen "en" sus instancias y que, por lo tanto, si no están instanciados no existen, y universales trascendentes o platónicos que no requieren de instanciaciones para existir (Cfr., Armstrong, 1978a:58-132; 1989:75-112). Correlativamente, los universales inmanentes son considerados entidades —en general— contingentes, pues, sólo existen en los mundos posibles en los que se encuentran instanciados, mientras que los universales trascendentes son considerados entidades necesarias, que existen de manera invariante en todos los mundos posibles (una excepción a esto en Tooley, 1987:113-120).

\footnotetext{
${ }^{3}$ Esta es la posición preferida por David Lewis. Los objetos posibles han de verse, en su teoría, como habitantes de mundos posibles que son entidades de la misma naturaleza que el mundo actual, pero un actualista podría acomodar la concepción lewisiana a su metafísica modal con objetos posibles apropiados, Cfr., Lewis (1983:12-19;1986:50-69).

${ }^{4}$ En la teoría defendida por Gonzalo Rodriguez-Pereyra, los objetos que son perfectamente semejantes pueden luego agruparse en una clase. Las funciones teóricas, sin embargo, dependen de la relación de semejanza y no de la existencia o inexistencia de clases de objetos (2002:50-52); la distinción con la teoría de Lewis en 56-62. Para una discusión de otras teorías nominalistas, Cfr., Armstrong (1978a:11-57).
} 
¿Cuál es, entonces, la distinción entre teorías de propiedades "escasas" y "abundantes"? Es difícil especificar aquí "cantidades" de propiedades, por lo que no tiene mucho sentido decir que en una concepción se postulan "más" propiedades que en la otra. La diferencia tiene que ver con las motivaciones teóricas que justifican la postulación de propiedades en nuestra ontología. Hay "roles" que se supone que deben cumplir las propiedades y que justifican su introducción en nuestras ontologías (Cfr., Lewis, 1986:56-63; Oliver, 1996:920). Ha sido puesto de relieve, en efecto, que estos "roles" parecen exigir candidatos de naturaleza muy diferente. Los mejores candidatos para satisfacer un tipo de rol teórico no son los mejores candidatos para satisfacer otros roles teóricos. Si se pone el énfasis en uno de estos tipos de roles se tienen propiedades "abundantes". Si se pone el énfasis en otro tipo de roles, en cambio, se tienen propiedades "escasas".

a) Propiedades "abundantes". Vienen dadas como el valor semántico de los predicados de un lenguaje. Como en un lenguaje natural cualquiera pueden formularse potencialmente infinitos predicados, estas propiedades también deben ser infinitas. Vienen a explicar por qué las oraciones en las que ocurre un predicado " $F$ " cualquiera tienen un valor de verdad determinado de acuerdo a la forma como tales oraciones se encuentran construidas. Debe haber, aquí, propiedades para predicados disyuntivos como "verzul" (esto es, verde si se es examinado antes del año $n$ o azul si se es examinado después del año $n$ ), pues las oraciones en las que esos predicados ocurren tienen valor de verdad y debe especificarse cómo es que tales predicados, entonces, efectúan una contribución sistemática a la determinación de ese valor de verdad. ${ }^{5}$

Íntimamente relacionado con lo anterior, las propiedades "abundantes" vienen a entregar contenidos para las actitudes proposicionales. Podemos definir una proposición, en efecto, como una propiedad 0-ádica, por lo que las operaciones de conformación de una propiedad — a partir de otras propiedadessirven, también, para explicar la estructura de una proposición. Por ejemplo, el estado intencional de creer que la estrella de la mañana es un cuerpo celeste puede diferenciarse del estado intencional de creer que la estrella de la tarde es un cuerpo celeste. En efecto, se pueden apreciar aquí dos proposiciones diferentes

(1) La estrella de la mañana es un cuerpo celeste

(2) La estrella de la tarde es un cuerpo celeste

\footnotetext{
${ }^{5}$ Algunas veces esta motivación se presenta como el problema de "lo uno sobre lo múltiple". Se trata de explicar por qué se pueden afirmar las oraciones " $\mathrm{F} a$ " y " $\mathrm{F} b$ " para objetos $a$ y $b$ diferentes entre sí. Esto es, se trata de explicar qué es lo que entrega un valor semántico al predicado "F" tal que es verdadero atribuirlo tanto al objeto $a$ como al objeto $b$.
} 
La diferencia entre (1) y (2) como propiedades 0 -ádicas tiene que ver con que, mientras (1) surge por la operación de inserción de "la estrella de la mañana" en la propiedad [ $\lambda x$ ( $x$ es un cuerpo celeste)], (2) surge por la operación de inserción de "la estrella de la tarde" en la misma propiedad. ${ }^{6}$ Cualquier sujeto racional que tenga un estado intencional de creencia cuyo objeto sea (1) estará tematizando una propiedad 0-ádica diferente de quien tenga un estado intencional tematizando (2). Así, las propiedades tendrán como condición de identidad las propiedades de que estén compuestas y el modo como hayan sido construidas mediante las operaciones pertinentes. En principio, las condiciones de identidad de estas propiedades "abundantes" deben ser tan finas como para discriminar tales diferentes estados intencionales. Si un sujeto racional, entonces, cree que hay un $\mathrm{P}$, pero no cree que hay un $\mathrm{Q}$, entonces $\mathrm{P} \neq \mathrm{Q}$. En general

(3) $\forall \forall \mathrm{P}_{1} \forall \mathrm{P}_{2}\left[\left(\mathrm{P}_{1}=\mathrm{P}_{2}\right) \leftrightarrow \forall \mathrm{S} \forall \mathrm{c}((\mathrm{S}\right.$ tiene en c el estado intencional $\Phi$ de que: $\left.\left[\ldots \mathrm{P}_{1} \ldots\right]\right) \leftrightarrow(\mathrm{S}$ tiene en $\mathrm{c}$ el estado intencional $\Phi$ de que: $\left.\left.\left.\left[\ldots \mathrm{P}_{1} / \mathrm{P}_{2} \ldots\right]\right)\right)\right]$

Aquí " $S$ " tiene como rango sujetos racionales y "c" tiene como rango contextos definidos como mundos posibles centrados. ${ }^{7}\left[\ldots \mathrm{P}_{1} \ldots\right]$ es una proposición donde ocurre la propiedad $\mathrm{P}_{1}$ y $\left[\ldots \mathrm{P}_{1} / \mathrm{P}_{2} \ldots\right]$ es la proposición que resulta de sustituir de manera uniforme todas las ocurrencias de $\mathrm{P}_{1}$ en $\left[\ldots \mathrm{P}_{1} \ldots\right]$ por $\mathrm{P}_{2}$.

b) Propiedades "escasas". Estas propiedades de entrada no están correlacionadas con los predicados de un lenguaje. No todos los predicados tendrán propiedades como sus valores semánticos y, por supuesto, no todas las propiedades serán el valor semántico de algún predicado. Aquí, la motivación para introducir propiedades no es de carácter semántico, ni tiene que ver con la necesidad de especificar los objetos de los estados intencionales. Las motivaciones centrales para introducir propiedades "escasas" tienen que ver con la explicación de respectos objetivos de semejanza, ${ }^{8}$ la explicación de la naturaleza de las

\footnotetext{
${ }^{6}$ En general, la operación de inserción (plug up) toma una propiedad $n$-ádica $\operatorname{Rx}_{1} \mathrm{x}_{2} \ldots \mathrm{x}_{\mathrm{n}} \mathrm{y}$ obtiene una propiedad $(n-1)$-ádica en donde la variable $j(1 \leq j \leq n)$ es sustituida por una constante de individuo, sea $\mathrm{Rx}_{1} \mathrm{x}_{2} \ldots a_{\mathrm{j}} \ldots \mathrm{x}_{\mathrm{n}}$. Para un desarrollo de este estilo de teoría de propiedades. ( $C f r$., por ejemplo, Parsons, 1980:63-97); el desarrollo de actitudes proposicionales en 108-123.

${ }^{7}$ Esto es, se trata de tríos ordenados $\langle\mathrm{w}, \mathrm{S}, \mathrm{t}\rangle$ en que $\mathrm{w}$ es un mundo posible, $\mathrm{S}$ es un sujeto racional seleccionado en ese mundo posible y $\mathrm{t}$ es un instante de tiempo. El mundo posible centrado selecciona, entonces, el estado mental de un sujeto racional en un instante de tiempo preciso en el que quedan fijados los estados intencionales de ese sujeto.

${ }^{8}$ El problema de "lo uno en lo múltiple" aquí es explicar cómo es que diferentes objetos pueden tener la misma naturaleza. No se trata, por lo tanto, simplemente de explicar cómo es que un predicado de algún lenguaje es atribuible a diferentes casos. Su punto de partida es la constatación de identidades específicas de una misma naturaleza en diferentes objetos (o eventos) sea que existan o no existan expresiones lingüísticas para ellas.
} 
relaciones causales y de las leyes naturales. Una propiedad es, aquí, un carácter, rasgo o respecto objetivo que determina la naturaleza intrínseca de las cosas y que determina las relaciones causales en las que un objeto pueda entrar. Estos rasgos, caracteres o respectos son los que típicamente deben entrar en las leyes naturales. Se trata, por lo tanto, de propiedades cuya existencia no puede ser descubierta por mera reflexión a priori, pues qué propiedades existan depende de qué respectos son realmente relevantes para especificar el desenvolvimiento de los procesos naturales. Esto es algo que se determina, al menos ordinariamente, mediante investigación empírica a posteriori. Es la ciencia y no algún filósofo quien debe decidir cuáles son realmente las propiedades en el mundo.

¿Qué propiedades existen en esta concepción? Algunos filósofos sostienen que existen aquellas propiedades sobre las que cuantifique la oración Ramsey que contenga la expresión de las leyes naturales del mundo y solamente ellas (Cfr., Mellor, 1995:185-199). Esto es, una vez que se da una descripción completa de cómo está constituido el mundo, se axiomatiza esta teoría de modo que garantice el mejor balance de informatividad y de poder deductivo. ${ }^{9}$ Las tesis, ya sean axiomas o teoremas de esta teoría, pueden ser tomadas como la expresión de las leyes naturales del mundo. Esta gran oración puede, luego, ser sometida a generalización existencial de segundo orden donde los lugares de predicado sean ligados por cuantificadores existenciales. Así, sea una oración máximamente descriptiva del mundo según los requerimientos indicados

(4) $\exists \mathrm{x}_{1} \exists \mathrm{x}_{2} \ldots \exists \mathrm{x}_{\mathrm{n}}\left(\mathrm{F}_{1} \mathrm{x}_{1} \mathrm{x}_{2} \ldots \mathrm{x}_{\mathrm{n}} \wedge \mathrm{F}_{2} \mathrm{x}_{2} \mathrm{x}_{3} \ldots \mathrm{x}_{\mathrm{n}-1} \wedge \ldots \wedge \mathrm{F}_{\mathrm{n}} \mathrm{x}_{\mathrm{n}} \mathrm{x}_{\mathrm{n}-1} \ldots \mathrm{x}_{1}\right)$

Esta oración (4) puede ser generalizada existencialmente con tal que resulte

(5) $\exists X_{1} \exists X_{2} \ldots \exists X_{n} \exists x_{1} \exists x_{2} \ldots \exists x_{n}\left(X_{1} x_{1} x_{2} \ldots x_{n} \wedge X_{2} x_{2} X_{3} \ldots x_{n-1} \wedge \ldots \wedge\right.$ $\left.\mathrm{X}_{\mathrm{n}} \mathrm{X}_{\mathrm{n}} \mathrm{X}_{\mathrm{n}-1} \ldots \mathrm{X}_{1}\right)$

Aquí, las variables $\mathrm{X}_{1}, \mathrm{X}_{2}, \ldots, \mathrm{X}_{\mathrm{n}}$ tienen como rango propiedades. La oración (5) dice que hay propiedades $X_{1}, X_{2} \ldots, X_{n}$ que se encuentran conectadas entre sí de cierta manera en el plexo de leyes naturales. Tales serán las propiedades existentes.

Otros autores no hacen apelación a las oraciones Ramsey, pero asumen un punto de vista básicamente semejante. Las leyes naturales son relaciones entre universales y, por lo tanto, deben suponerse como existentes aquellos universales que sean exigidos por tales leyes (Cfr., Armstrong, 1983:75-173; Dretske, 1977;

\footnotetext{
${ }^{9}$ Esta concepción de las leyes naturales es la defendida por David Lewis (1973: 72-77). Se trata de una forma de teoría de la regularidad que no es, precisamente, el tipo de teoría que ha sido tomada como apoyando la existencia de universales "escasos", pero se expone aquí simplemente para facilitar la exposición.
} 
Tooley, 1977;1987:37-169). Las relaciones causales son normalmente postuladas como teniendo por relata a eventos. Ordinariamente un evento queda especificado por una propiedad, un objeto y un lapso temporal (intuitivamente, el lapso de tiempo en que ese objeto posee tal propiedad). Existirán las propiedades que sean relevantes para determinar las relaciones causales objetivas entre eventos. ${ }^{10}$ Como, al menos ordinariamente, las relaciones causales estarán determinadas por leyes naturales, las propiedades que sean exigidas por las leyes naturales coincidirán con las propiedades que sean exigidas por las conexiones causales objetivas. Estas mismas propiedades son las que deberían aparecer en una oración Ramsey que exprese adecuadamente las leyes naturales existentes en un mundo.

Lo más usual ha sido aquí postular, por lo tanto, condiciones de identidad conectadas a los poderes causales de que una propiedad inviste a sus poseedores (Cfr., Shoemaker, 1980). El "poder causal" asociado con una propiedad $\mathrm{P}$ es el conjunto de conexiones causales en las que puede intervenir un objeto, dado que posee la propiedad P. Sea C la relación $n$-ádica de causar algo (convendrá tomarla como una relación multígrada para preservar la generalidad de la formulación). El poder causal de un objeto $\mathrm{x}$ que posee una propiedad $\mathrm{P}$ en el instante de tiempo $\mathrm{t}$ puede ser especificado como

$$
\diamond \exists \mathrm{y}_{1} \exists \mathrm{y}_{2} \ldots \exists \mathrm{y}_{\mathrm{n}}\left([\mathrm{Pxt}] C \mathrm{y}_{1} \mathrm{y}_{2} \ldots \mathrm{y}_{\mathrm{n}}\right) \wedge \diamond \exists \mathrm{y}_{1} \exists \mathrm{y}_{2} \ldots \exists \mathrm{y}_{\mathrm{n}}\left(\mathrm{y}_{1} \mathrm{y}_{2} \ldots \mathrm{y}_{\mathrm{n}} C[\mathrm{Pxt}]\right)
$$

Aquí en (6) [Pxt] designa el evento de instanciar el objeto $\mathrm{x}$ la propiedad $\mathrm{P}$ en el instante de tiempo $t$. Las variables $\mathrm{y}_{1}, \mathrm{y}_{2}, \ldots, \mathrm{y}_{\mathrm{n}}$ tienen como rango a eventos. En general, las condiciones de identidad de un evento están fijadas por un objeto, una propiedad poseída por ese objeto y un instante de tiempo. El "poder causal" que entrega una propiedad es aquello que podría ser causado por el evento de estar esa propiedad instanciada por un objeto en un instante de tiempo y por aquello que podría causar que esa propiedad estuviese instanciada en un objeto en un instante de tiempo. Pues bien, las condiciones de identidad para propiedades "escasas" resultarían las siguientes

$$
\begin{aligned}
& \text { (7) } \forall \mathrm{P}_{1} \forall \mathrm{P}_{2}\left[( \mathrm { P } _ { 1 } = \mathrm { P } _ { 2 } ) \leftrightarrow \forall \mathrm { x } \forall \mathrm { t } \diamond \exists \mathrm { y } _ { 1 } \exists \mathrm { y } _ { 2 } \ldots \exists \mathrm { y } _ { \mathrm { n } } \quad \left(\left(\left(\left[\mathrm{P}_{1} \mathrm{xt}\right] C \mathrm{Cy}_{1} \ldots \mathrm{y}_{\mathrm{n}-1}\right) \wedge\right.\right.\right. \\
& \left.\left.\left(\mathrm{y}_{2} \ldots \mathrm{y}_{\mathrm{n}} \mathrm{C}\left[\mathrm{P}_{1} \mathrm{xt}\right]\right)\right) \leftrightarrow\left(\left(\left[\mathrm{P}_{2} \mathrm{xt}\right] C \mathrm{C}_{1} \ldots \mathrm{y}_{\mathrm{n}-1}\right) \wedge\left(\mathrm{y}_{2} \ldots \mathrm{y}_{\mathrm{n}} \mathrm{C}\left[\mathrm{P}_{2} \mathrm{xt}\right]\right)\right)\right]
\end{aligned}
$$

esto es, las propiedades $\mathrm{P}_{1}$ y $\mathrm{P}_{2}$ son idénticas si y sólo si confieren los mismos poderes causales. Aquí, en general, vale que [ $\forall \mathrm{P} \forall \mathrm{x} \forall \mathrm{t}$ (Pxt $\leftrightarrow([$ Pxt] existe))].

\footnotetext{
${ }^{10}$ Para una explicación más detallada de las variaciones terminológicas que pueden encontrarse aquí, Cfr., Oliver (1996:17-18). Algunos autores hablan de "situaciones", "estados de cosas", "hechos", incluso "facta". Las condiciones de identidad en todos estos casos son, básicamente, las mismas.
} 


\section{UNIVERSALES INMANENTES ESCASOS Y UNIVERSALES TRASCENDENTES "ABUNDANTES"}

Ha sido usual sostener que si han de admitirse propiedades "abundantes" y si las propiedades han de tomarse como universales, entonces deben tomarse tales universales como trascendentes. También ha sido usual sostener que si han de admitirse propiedades universales "escasas", entonces deben tomarse tales universales como inmanentes. En el caso de las propiedades "abundantes", la conexión con los universales trascendentes parece bien razonable. En efecto, aquí se trata de especificar los valores semánticos para todos los predicados de cualquier lenguaje y de especificar los contenidos de todo estado intencional posible. Es obvio que hay oraciones en las que aparecen términos que no parecen tener referencia, ya sean nombres propios, descripciones vacías o sencillamente predicados que nada satisfacen, como

(8) Los centauros del monte asolaron de nuevo las viñas

donde hay una diferencia bastante marcada entre esta oración (8) y

(9) Los aardvags del monte asolaron de nuevo las viñas

La diferencia estriba en que existe el predicado "ser un centauro" en nuestro lenguaje, pero no existe el predicado "ser un aardvag". Del mismo modo, alguien podría tener el estado intencional de creer que los centauros del monte asolaron las viñas, pero no tener el estado intencional de creer que los aardvags del monte asolaron las viñas, como tampoco creer que los trolls del monte asolaron las viñas. ${ }^{11}$ Cualquiera sea la explicación de los valores semánticos de tales predicados y de los contenidos de los diferentes estados intencionales, esta explicación tendrá que postular algún tipo de entidades que no se encuentran instanciadas en el mundo actual o que, sencillamente, no son entidades físicas interactuando físicamente con otras entidades del mundo actual. Un universal trascendente parece un buen candidato para asumir los roles exigidos a las propiedades "abundantes". Algunos de los universales no están instanciados actualmente pero, otros sí, por lo que puede suponerse que nuestro acceso epistemológico a esas entidades no exige de nuestra parte capacidades cognitivas exorbitantes. Esto parece una ventaja respecto de, por ejemplo, la postulación de objetos inexistentes meinongianos.

\footnotetext{
${ }^{11}$ En efecto, uno podría sentirse inclinado a decir que los predicados "ser un centauro", "ser un aardvag" y "ser un troll" son todos vacíos, por lo que nada los satisface. Todas las oraciones en las que aparezcan tales predicados tendrán que ser por igual falsas o carentes de valor de verdad. Esto no permitiría discriminar entre estados intencionales en que aparece el contenido "centauro" y el contenido "troll", cuando intuitivamente tal diferencia existe y es relevante. Parece necesario asignarle un valor semántico distinto a unos y otros predicados.
} 
En el caso de las propiedades "escasas", en cambio, pareciera que se requirieran entidades que han de postularse como integrando el tráfago de las transacciones causales, algo que se encuentre físicamente localizado en el espacio y en el tiempo, capaz de actuar y de padecer la acción. En efecto, las propiedades "escasas" son postuladas, precisamente, porque se supone que debe haber propiedades como constituyentes ontológicos de los eventos que son los relata de las relaciones causales. Se supone, también, que cuando la ciencia natural describe los respectos que son relevantes para determinar por qué los procesos físicos se desarrollan de la forma en que lo hacen, es porque selecciona las propiedades que han de entrar en las leyes naturales que son, por lo demás, conexiones nómicas entre universales o, bien, simplemente universales. Esos respectos son los que también especifican la semejanza o desemejanza objetiva entre entidades que poseen o no poseen la misma naturaleza. No es extraño que los universales inmanentes hayan sido considerados excelentes candidatos para asumir estos roles, pues, existen sólo si tienen instancias, se encuentran en sus instancias y, además, en el lugar y en el tiempo donde tales instancias están ubicadas. ${ }^{12}$ Estas intuiciones han sido formuladas como el "principio eleático" por Armstrong: "todo lo que existe hace una diferencia en los poderes causales de algo" (Armstrong, 1997:41). Un universal puede ser admitido en nuestra ontología sólo si es que su introducción modifica el patrón de eventos físicos del mundo, sea porque aumenta o disminuye el poder causal de algo que posee tal propiedad. Pareciera, aquí, que un universal trascendente no es apto para cumplir estas funciones.

\section{UNIVERSALES TRASCENDENTES "ESCASOS"}

Tal como se ha indicado, entonces, se tiende a pensar que los roles que le son asignados a las propiedades "abundantes" han de corresponder a universales trascendentes, mientras que los roles que le son asignados a las propiedades "escasas" han de corresponder a universales inmanentes. Las condiciones de identidad de los universales trascendentes, por lo tanto, deberían corresponder a las indicadas por el principio (3), mientras que las condiciones de identidad de los universales inmanentes deberían corresponder a las indicadas por el principio (7). Unos son identificados por su función para especificar actitudes intencionales, mientras que los otros son identificados por su función en la asignación de poderes causales.

\footnotetext{
${ }^{12}$ Lo mismo podría decirse, naturalmente, de tropos "escasos". Las clases naturales de Lewis y las relaciones de semejanza perfecta primitiva del nominalista de semejanza se supone que también satisfacen los mismos roles, o roles análogos, mediante entidades que están en el mundo físico.
} 
Contra lo que pudiera suponerse, sin embargo, los universales trascendentes también pueden tener condiciones de identidad de acuerdo a lo asignado en el principio (7). También es posible postular universales no instanciados de carácter "escaso", ligados a lo que muestre la investigación empírica a posteriori. Esto, por supuesto, no es afirmar que los universales trascendentes tienen, de hecho, tales condiciones de identidad. Lo que se pretende, aquí, es simplemente mostrar que no es incoherente tal suposición. Un primer elemento a tener en consideración es que una de las motivaciones para aceptar universales "escasos", la concepción de las leyes naturales como relaciones entre universales, parece apuntar hacia la existencia de universales trascendentes. ${ }^{13}$ Tooley ha presentado una serie de casos en los que pareciera que debe aceptarse la existencia de una ley natural, aun cuando los universales que aparecen ahí no están instanciados (Cfr., Tooley, 1987:67-75;113120). Los casos presentados por Tooley pueden ser generalizados. Supóngase un mundo posible $\mathrm{w}_{1}$ en donde se hubiesen descubierto cinco y solamente cinco partículas fundamentales. En todos los casos se han hallado leyes naturales que determinan el comportamiento de estas partículas entre sí, con la única excepción de las partículas de tipo D y las partículas de tipo E. Las leyes que han sido descubiertas para la interacción de los restantes tipos de partículas son de carácter primitivo, no pudiendo deducirse de otras leyes de carácter más básico o de carácter más general. Supóngase también que, de acuerdo a las condiciones de contorno de $\mathrm{w}_{1}$, nunca entrarán en interacción las partículas de tipo D con las partículas de tipo E. En este caso, parece razonable sostener que hay una ley natural que determina las interacciones entre partículas D y E, aunque no sea cognoscible. Si existe la ley natural en cuestión, deben existir los universales que están ahí conectados nomológicamente, por lo que pareciera que debemos admitir la existencia de un universal relacional estar una partícula $D$ en interacción con una partícula $E$, aun cuando ese universal no se encuentra instanciado.

Un caso más sencillo es el siguiente: en el mundo actual $\mathrm{w}_{\mathrm{A}}$ es una ley natural que los objetos con carga eléctrica negativa atraen a objetos con carga eléctrica positiva. Como es verdadera esta ley natural, es verdadero en $\mathrm{w}_{\mathrm{A}}$ el condicional contrafáctico

Si hubiese un objeto con carga eléctrica negativa, atraería a los objetos con carga eléctrica positiva.

\footnotetext{
${ }^{13}$ Hay otras concepciones en las, rechazándose también las concepciones humanas de las leyes como regularidades, se las ve no como relaciones de necesitación contingente entre universales, sino como esenciales a las propiedades que conectan (Cfr., Ellis, 2001:201-228; Mumford, 2004:125-205; Bird, 2007:43-65;189-203; Swoyer, 1982; Molnar, 2003: 199). En estas concepciones, los casos de tipo Tooley también apuntan hacia universales no instanciados.
} 
Considérese ahora un mundo posible $\mathrm{w}_{2}$ en el que no existan objetos con carga eléctrica negativa. Parece razonable pensar que el condicional contrafáctico (10) también es verdadero en $\mathrm{w}_{2}$, pues, aun cuando no hay objetos con carga eléctrica negativa en $\mathrm{w}_{2}$, si los hubiese, estos objetos atraerían a los objetos con carga eléctrica positiva. Si es verdadero el condicional (10), parece razonable sostener que también existe en $\mathrm{w}_{2}$ la misma ley natural que en $\mathrm{w}_{\mathrm{A}}$. Pero si existe la ley, deben existir también los universales que están conectados por ella, aun cuando no estén instanciados en $\mathrm{w}_{2}$. Luego, parecen haber universales no instanciados.

No es necesario aquí discutir en detalle estos casos y las maniobras que han sido desplegadas para neutralizarlos por los defensores de los universales inmanentes. Lo que interesa considerar, aquí, es cómo en estos casos se está apelando a una línea de argumentación característica para justificar la existencia de propiedades "escasas", pero que no conduciría a la aceptación de universales inmanentes, sino de universales trascendentes. ¿Cómo es esto posible? De acuerdo al principio (7) dos propiedades son idénticas si y sólo si los poderes causales que confieren a sus poseedores son también idénticos. Estos poderes causales quedan, a su vez, especificados por el conjunto de todas las conexiones causales en las que el objeto que posee tal propiedad podría estar envuelto, dado que posee tal propiedad. La duda central que surge cuando se trata de aplicar este principio de identidad a universales trascendentes es que no se ve cómo es que un universal de este tipo puede conferir un poder causal en el objeto que lo instancia. Si se trata de un universal inmanente o de un tropo, en cambio, parece claro que es la propiedad misma la que está envuelta en relaciones causales, pues, es un integrante intrínseco de los eventos los que son, a su vez, los relata directos de la conexión causal. Un universal trascendente, sin embargo, no es integrante intrínseco de un evento.

El punto es que un universal trascendente confiere poderes causales en los objetos en los que está instanciado debido a que los tropos de tal universal son componentes intrínsecos de los eventos en los que tal objeto es integrante y que son los relata directos de las conexiones causales. En efecto, una ontología que postule universales trascendentes debe, también, postular tropos, pues las instanciaciones del universal en un objeto particular (o entidad particular) son precisamente eso. Nadie pone en cuestión que un tropo interviene en relaciones causales, luego, un objeto tiene los poderes causales que tiene debido a que instancia tales y cuales universales. Un universal, sea trascendente o inmanente, por tanto, confiere poderes causales en los objetos en los que se encuentra instanciado, debido a que un objeto se verá envuelto en determinadas interconexiones causales dados los universales que se encuentre instanciando. Si un universal, entonces, confiere poderes causales, sus condiciones de identidad pueden ser especificadas mediante tales poderes de acuerdo al principio (7). 
Esta solución simple al problema puede parecer sospechosa para algunos. La sospecha surge porque parece no económico postular universales y, además, tropos. ${ }^{14}$ Hay defensores de universales que, por eso, no requieren tropos. Hay defensores de tropos que pretenden eliminar mediante ellos a los universales. Una posición que requiera tanto a los universales como a los tropos parece combinar todos los costos ontológicos. El problema, aquí, es que la necesidad de postular tropos no es una peculiaridad, en realidad, de las teorías de universales trascendentes. Si se postulan universales inmanentes, también es necesario postular la instanciación particular del universal en un particular. En efecto, tal como ha sido puesto de relieve con frecuencia, un estado de cosas no es simplemente un listado de un particular (o de varios particulares) y un universal. El listado es ontológicamente inerte si es que el universal y el particular están desconectados entre sí. Tampoco puede pensarse en el estado de cosas como una $n$-tupla, esto es, como una entidad conjuntista, pues las entidades conjuntistas vienen garantizadas no sólo para los universales y particulares que configuran, de hecho, estados de cosas, sino también para los universales y particulares que no configuran estados de cosas (e, incluso, para universales y particulares que no pueden configurar un estado de cosas). Si se quiere decir, por otro lado, que particular y universal "componen" el estado de cosas, es una forma de composición nomereológica (Cfr., Armstrong, 1997:119-123). También se ha pretendido decir que la conexión entre universal y particular para configurar estados de cosas es un "vínculo" no relacional. Sin embargo, hay motivaciones independientes para esta tesis y es muy extraño hablar de una relación que no es relación. Si hay una diferencia ontológicamente sustantiva entre un estado de cosas y el mero listado de los particulares y universales que lo componen, entonces, debe haber una instanciación de ese universal en ese particular. Llámesela como se la llame, la instanciación particular de un universal es un tropo.

Resulta entonces que las teorías de universales inmanentes no están realmente en una posición sustantivamente mejor que las teorías de universales trascendentes. Si tanto en uno como en otro caso deben postularse tropos junto con universales, entonces no debe causar extrañeza que los tropos de un universal cumplan una función relevante para que el universal confiera poderes causales. Dado que el universal confiere poderes causales, nada parece impedir que esos

\footnotetext{
${ }^{14}$ Por de pronto, esto sería una dificultad cualquiera sean las condiciones de identidad que se postulen para los universales trascendentes. Una formulación de esta "sospecha" en Armstrong, (1989:132-133).
} 
poderes causales sean tomados como fijando las condiciones de identidad de tales universales.

\author{
Pontificia Universidad Católica de Valparaíso* \\ Instituto de Filosofía \\ Avda. El Bosque 1290, Viña del Mar (Chile) \\ jose.alvarado.m@ucv.cl
}

\title{
BIBLIOGRAFÍA
}

Armstrong, David M. A World of States of Affairs. Cambridge: Cambridge University Press, 1997.

------ Universals. An Opinionated Introduction. Boulder: Westview, 1989.

------ What is a Law of Nature? Cambridge: Cambridge University Press, 1983.

------ Universals and Scientific Realism. Volumen I. Nominalism and Realism. Cambridge: Cambridge University Press, 1978a.

------ Universals and Scientific Realism. Volume II. A Theory of Universals. Cambridge: Cambridge University Press, $1978 b$.

Bird, Alexander. Nature's Metaphysics. Oxford: Clarendon Press, 2007.

Campbell, Keith. "The Metaphysic of Abstract Particulars", en Midwest Studies in Philosophy 6. 1981:477-488.

Drestke, Fred. "Laws of Nature", en Philosophy of Science 44. (1977):248-268.

Ellis, Brian. Scientific Essentialism. Cambridge: Cambridge University Press.

Lewis, David (1973), Counterfactuals. Oxford: Blackwell, 2001.

------ "New Work for a Theory of Universals", en Australasian Journal of Philosophy 61. 1983:343-377. Reimpreso en Lewis (1999):1-55. Se cita por esta última versión.

------ Papers in Metaphysics and Epistemology. Cambridge: Cambridge University Press, 1999.

------ On the Plurality of Worlds. Oxford: Blackwell, 1986.

Mellor, Hugh. The Facts of Causation. London: Routledge, 1995.

Molnar, George. Powers. A Study in Metaphysics. Oxford: Oxford University Press, 2003.

Mumford, Stephen. Laws in Nature. London: Routledge, 2004.

Oliver, Alexander. "The Metaphysics of Properties", en Mind 105, (1996):1-80.

Parsons, Terence. Nonexistent Objects. New Haven: Yale University Press, 1980.

Rodriguez-Pereyra, Gonzalo. Resemblance Nominalism. A Solution to the Problem of Universals. Oxford: Clarendon Press, 2002.

Shoemaker, Sydney. "Causality and Properties", en P. van Inwagen (Ed.). Time and Cause. Dordrecht: Reidel, 1980:109-135. Reimpreso en Identity, Cause, and Mind. Philosophical Essays. Oxford: Clarendon Press, 2003:206-233. Se cita por esta última versión. 
José Tomás Alvarado Marambio

Swoyer, Chris. "The Nature of Natural Laws", en Australasian Journal of Philosophy 60. 1982:203-223.

Tooley, Michael. "The Nature of Laws", en Canadian Journal of Philosophy 74. 1977:667-698.

------ Causation. A Realist Approach. Oxford: Clarendon Press, 1987. 